\title{
COMPARISON OF AODV AND ANTHOCNET IN STATIC WIRELESS NETWORK
}

\author{
R. Anusha ${ }^{1}$ and Dr. P. Chenna Reddy ${ }^{2}$ \\ ${ }^{1}$ Computer Science and Engineering, JNTUA College of Engineering, Pulivendula \\ ${ }^{2}$ Professor of CSE Department, JNTUA College of Engineering, Pulivendula
}

\begin{abstract}
$A O D V$ is an on-demand reactive protocol which is on the standardization process of Internet engineering task force. This protocol initiates route discovery process when route is required to send packets from sender to receiver. AntHocNet depends on Ant Colony Optimization technique and is considered as hybrid routing protocol, which consists of reactive path setup and proactive path management. In ACO routing algorithm ants move between nest and the food source by laying pheromone trails to collect routing information. This paper does the performance comparison of protocols AODV and AntHocNet in static wireless networks. The performance is analyzed by metrics Packet Delivery Ratio, End-to-End Delay, Loss Rate, Throughput and Jitter. Routing protocols are evaluated by using User Datagram Protocol as transport protocol, Network Simulator (NS2.34) and by using 802.11 and 802.11b.
\end{abstract}

\section{KEYWORDS}

AODV, AntHocNet, ACO, performance.

\section{INTRODUCTION}

The original standard IEEE 802.11 provides data rates $1 \mathrm{Mbps}$ and $2 \mathrm{Mbps}$ and a group of fundamental signaling and other services. The most critical issue effecting WLAN demand has been limited throughputs. The IEEE is rectified as $802.11 \mathrm{~b}$ to support transmission of large data rates of $11 \mathrm{Mbps}$. AODV routing design is intended to use in a wireless network. It is on the standardization process and is the widely used routing protocol in ad hoc networks.

Ant colony optimization (ACO) routing algorithm is derived from swarm intelligence [1]. It overcomes the complex problems by cooperation of simple ants. Individual ants communicate indirectly by modifying their environment instead of direct communication. Ants collect routing message repeatedly by sampling of multiple paths. Ant's starts from sender to receiver by collecting quality of paths information and return back to destination to update routing information at intermediate nodes. Information about routing can update by considering sample paths. A chemical substance called pheromone is downed by the ants while travelling.

AntHocNet protocol consists of reactive route set up phase and proactive path management [2]. The reactive phase collects routes information about destinations which are participating in communication session. At the start of a communication session, the sender checks its corresponding pheromone table to check if it has any routing information available for the requested destination. If no route is identified then it initiates a reactive process to identify the first route to the receiver by sending an ant packet out over the network and the type of ant packet is reactive forward ant (RFA). When a node has routing message about the ant destination in its pheromone table then RFA is unicasted, otherwise broadcasted.

DOI: $10.5121 /$ ijci.2015.4210 
International Journal on Cybernetics \& Informatics (IJCI) Vol. 4, No. 2, April 2015

RFAs maintain the visited nodes information on their way to the destination [2]. RFA is replaced as reactive backward ant (RBA) when it reaches the destination. Destination considers the first RFA, while subsequent copies are destroyed. RBA follows the path traced by RFA. On its way, it collects quality information about links of the path, updating of routing tables at each node and at the source based on this quality information. This way, a route between transmitter and receiver is established at completion of the reactive path setup process.

Once the route is compiled via the reactive path setup process, then the execution of the proactive route maintenance process is initiated and gets to update, extend and improve the available routing information [2]. This process continues using pheromone diffusion process and proactive ant sampling process. The purpose of the pheromone diffusion sub process is to spread out pheromone message which is stored by the ants. If best pheromone is available then nodes will periodically broadcast messages.

Link failure can be identified when the hello message is not received in few seconds or when unicasted transmission to neighbor fails [2]. Then the link failure notification message is sent to all its neighbors and corresponding pheromone tables are updated. Local route repair process starts when link failure is identified while transmitting data packets. Repair forward ants are broadcasted when no routing information is available. It acts similar to reactive forward ant but only difference is repair forward ant is broadcasted only over a limited number of hops.

When repair forward ant reaches destination then it is converted into repair backward ant [2]. Repair backward ant acts exactly like reactive backward ant to reach source. When it reaches source data packet is sent to destination.

\section{LITERATURE SURVEY}

Several kinds of researchers have studied and analyzed various routing protocols taking into observation different metrics as basis for performance evaluation. Gianni di Caro, Frederick Ducatelle, Luca Maria Gambardella [3], explained AntHocNet protocol inspired by ACO routing algorithm. The protocol contains both proactive and reactive elements. In the series of simulation evaluations the paper shows that AntHocNet has high performance over AODV in case of metrics Packet delivery ratio, average End-to-End Delay and Jitter. For metric Routing Overhead AntHocNet protocol is inferior to AODV.

Maahi Amit Khemchandani, Prof. B. W. Balkhande [4], published a paper on comparison of routing protocols AntHocNet, AODV and DSR. The simulation leads that AntHocNet performance is more than AODV and DSR routing protocols in case of metrics Loss packet ratio at high rates, at many of nodes and with high mobility. In terms of normalized routing overhead AntHocNet performance is inferior to DSR at lower rates. Finally the paper concludes that AntHocNet is suggested for high scale networks with increased data rates and mobility. At increased data rates AODV's and DSR's performance either decreases or very low but AntHocNet performance is either constant or increases.

Annapurna P Patil, K Rajanikant, Rakshith H P [5], discussed the implementation as well as analyzed the performance of AntHocNet algorithm. The paper evaluates the protocol AntHocNet and AODV by comparing using QualNet.

Ahmed M. Abd Elmoniem, Hosny M. Ibrahim, Marghny H. Mohamed, Abdel-Rahman Hedar [6], proposed two alternative protocols like Multi-Route AODV Ant routing (MRAA) and modified with Load Balanced Multi-Route AODV Ant routing algorithm (LBMRAA) to use ACO technique and also to identify multiple paths from sender to receiver. Simulation results shows 
that proposed MRAA protocol yields best performance than AODV and the modified load balancing protocol LBMRAA performs better than MRAA.

S.B. Wankhade and M.S. Ali [1] have done investigation on Ant routing protocols for MANETs. The paper concludes that existing routing algorithms are unsuitable for routing after failure of link because of node mobility, unlimited links and limited resources in MANET. So this problem is overcome by ant group based algorithm that uses Fuzzy rule-based systems.

D. Sivakumar, B. Suganthi [7], examines the performance of routing protocols AntHocNet, AOMDV and AODV. The metrics PDR, Delay, Throughput and Loss Rate are evaluated by varying number of nodes. By comparing routing protocols the performance of AntHocNet is higher than other protocols AOMDV and AODV. AntHocNet protocol effectively utilizes the reactive and proactive routing methods.

Nishitha Taraka, Amarnath Emani, [8], presented that routing problem in ad hoc network can be solved by using Ant Colony Optimization algorithm. Simulation results demonstrate that AntHocNet protocol is performed better than other protocols AODV and DSR at high data rates as well as at large number of nodes, whereas at low data rates and at less number of nodes the performance of AntHocNet is lower than AODV and DSR. So the paper suggested that AntHocNet protocol is suitable at high data rates or a high number of nodes.

N. S. Labhade, S. S. Vasekar [9], compares the performance of different routing protocols like DSDV, AODV, and AntHocNet. Metrics End-to-End delay and Throughput are evaluated at different number of nodes and simulation times. Simulation results showed that, AntHocNet protocol had low End-to-End delay and high Throughput than AODV and DSDV. The performance of AntHocNet is better than other protocols AODV and DSDV.

\section{SimUlation}

\subsection{Simulation Environment}

In this paper static wireless network is considered. The topology contains nodes which are placed at fixed positions as shown in Figure 1. In topology, two nodes are considered as source and two nodes are considered as destinations. The distance between nodes is $200 \mathrm{~m}$ and packet size is 210 bytes for UDP. Simulation time is $100 \mathrm{sec}$. For UDP Constant bit rate (CBR) is considered.

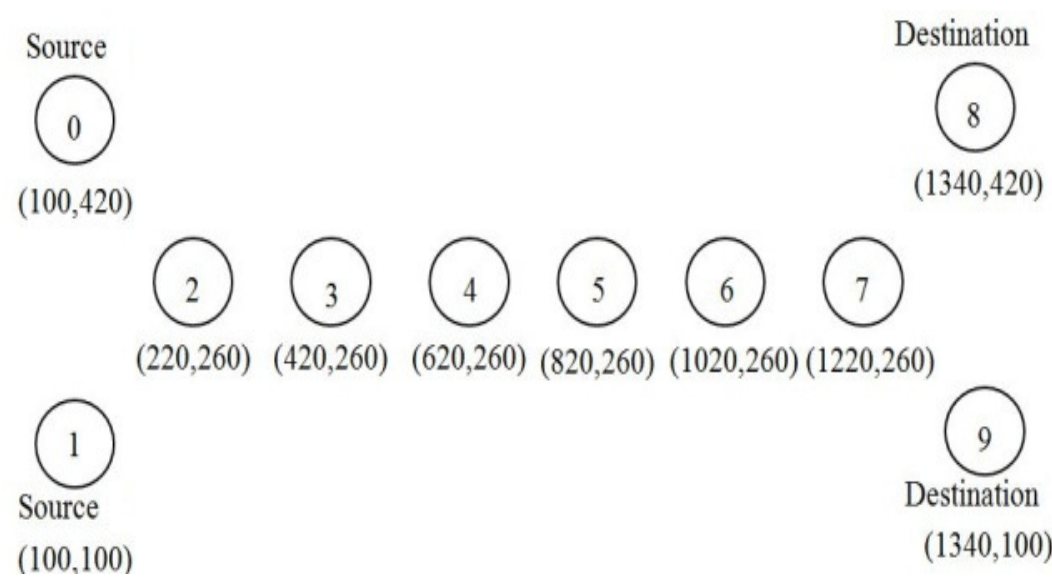

Figure 1: Position of nodes in wireless topology 


\subsection{Metrics}

The metrics that are used for comparing the AntHocNet and AODV protocols are Throughput, Loss Rate, End-to-End delay, Jitter and Packet Delivery Ratio.

- Throughput: Throughput is the rate of receiving data by the server and it is rated as bits per second (bits/sec).

- Packet Loss Rate: Loss Rate is the ratio of packets dropped to the sent packets from sender.

- End-to-End delay: End-to-End delay is the average time taken by a data packet to arrive at the receiver $(\mathrm{sec})$.

- Jitter: Jitter is the difference in delay at inter-arrival of packets $(\mathrm{sec})$.

- Packet Delivery Ratio: It refers the ratio of total packets received to the total packets sent.

\subsection{Simulation Results and Analysis}

UDP is the transport protocol, attached at source nodes. CBR is the constant bit rate, attached at both the source nodes to generate traffic. The data rate is fixed throughout simulation time. The data of both the sources is same. The Simulation results are explained in two cases. In first case Mac Layer is 802.11 and metrics Loss Rate, Packet Delivery Ratio, End-End Delay, Throughput and Jitter are evaluated at different data rates. In second case Mac Layer is 802.11b and same metrics are evaluated at data rates.

\subsubsection{Case 1: 802.11}

Table 1. UDP Loss Rate with variation in data rates

\begin{tabular}{|l|l|l|}
\hline \multirow{2}{*}{$\begin{array}{l}\text { Data Rate } \\
\text { Mbps) }\end{array}$} & Loss Rate \\
\cline { 2 - 3 } & AODV & AntHocNet \\
\hline 1 & 0.967 & 0.973 \\
\hline 2 & 0.983 & 0.986 \\
\hline 3 & 0.988 & 0.99 \\
\hline 4 & 0.991 & 0.997 \\
\hline 5 & 0.993 & 0.995 \\
\hline 6 & 0.994 & 0.995 \\
\hline 7 & 0.995 & 0.996 \\
\hline 8 & 0.996 & 0.997 \\
\hline
\end{tabular}


International Journal on Cybernetics \& Informatics (IJCI) Vol. 4, No. 2, April 2015

Table 2. UDP PDR with variation in data rates

\begin{tabular}{|l|l|l|}
\hline \multirow{2}{*}{$\begin{array}{l}\text { Data Rate } \\
\text { Mbps) }\end{array}$} & PDR & AntHocNet \\
\cline { 2 - 3 } & AODV & 0.027 \\
\hline 1 & 0.033 & 0.014 \\
\hline 2 & 0.017 & 0.009 \\
\hline 3 & 0.011 & 0.0026 \\
\hline 4 & 0.0085 & 0.0051 \\
\hline 5 & 0.0067 & 0.0049 \\
\hline 6 & 0.0056 & 0.0041 \\
\hline 7 & 0.0049 & 0.00301 \\
\hline 8 & 0.0042 & \\
\hline
\end{tabular}

Table 3. UDP End-to-End delay with different variation in data rates

\begin{tabular}{|l|l|l|}
\hline \multirow{2}{*}{$\begin{array}{l}\text { Data Rate } \\
\text { Mbps) }\end{array}$} & End-to-End Delay (sec) \\
\cline { 2 - 3 } & AODV & AntHocNet \\
\hline 1 & 3.026 & 3.12 \\
\hline 2 & 2.416 & 2.96 \\
\hline 3 & 2.696 & 3.13 \\
\hline 4 & 2.3007 & 3.15 \\
\hline 5 & 2.175 & 2.92 \\
\hline 6 & 2.138 & 2.135 \\
\hline 7 & 2.201 & 3.164 \\
\hline 8 & 2.529 & 3.142 \\
\hline
\end{tabular}

\subsubsection{Graphs for Throughput}

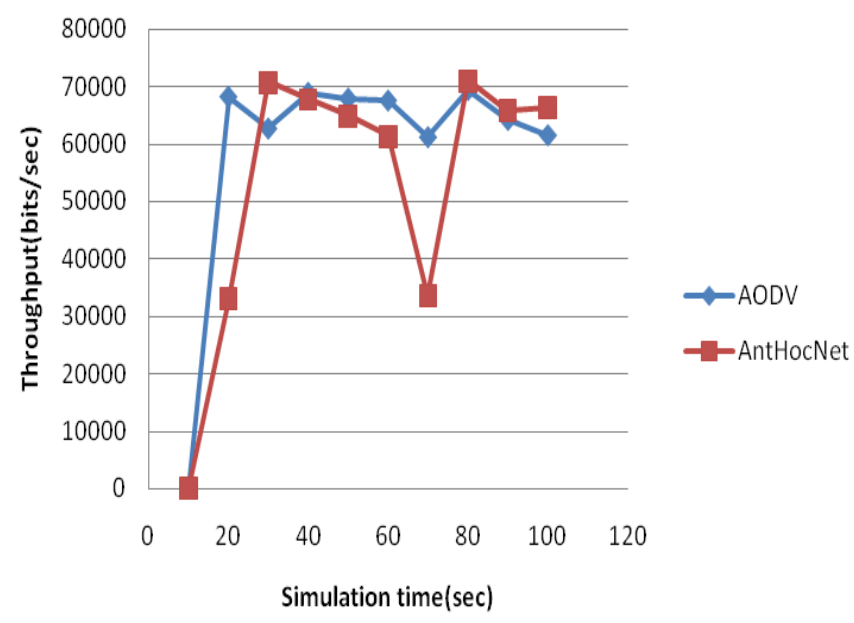

Figure 2. UDP Throughput at Data Rate 1Mbps 
International Journal on Cybernetics \& Informatics (IJCI) Vol. 4, No. 2, April 2015

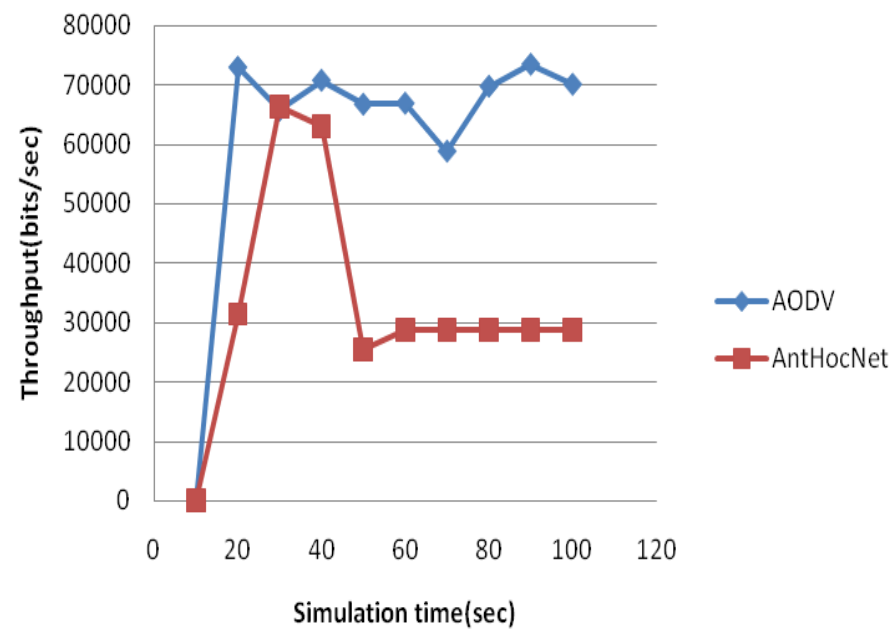

Figure 3. UDP Throughput at Data Rate 4Mbps

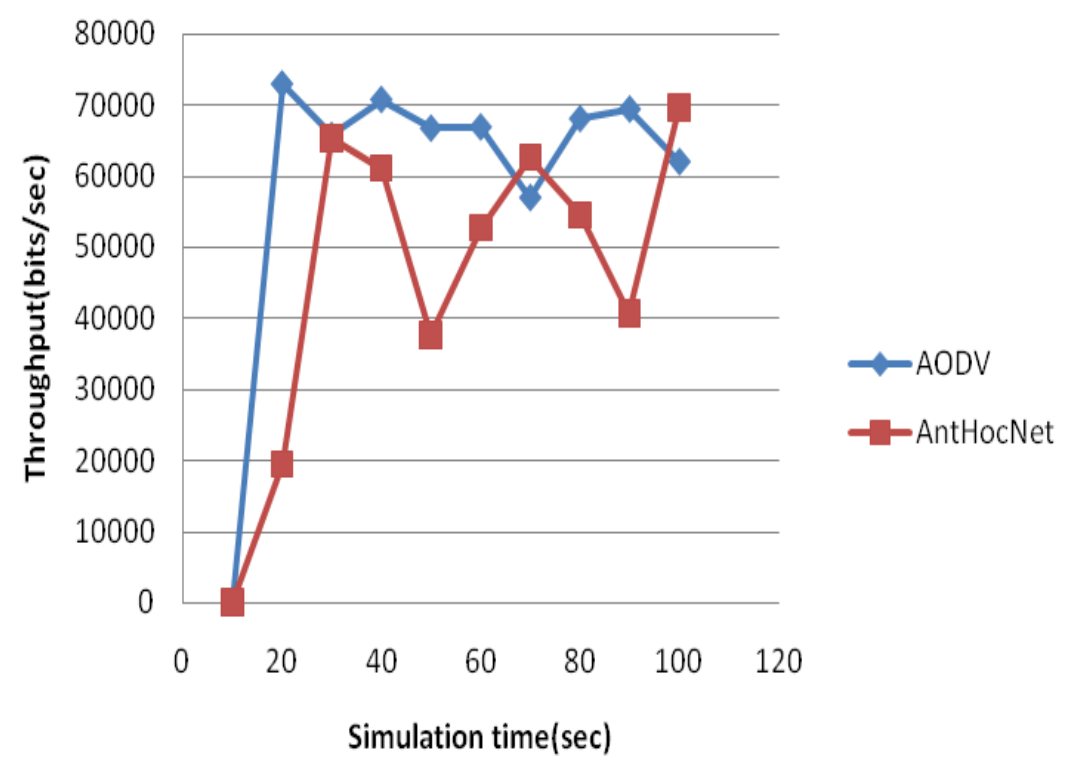

Figure 4. UDP Throughput at Data Rate $8 \mathrm{Mbps}$ 
International Journal on Cybernetics \& Informatics (IJCI) Vol. 4, No. 2, April 2015

\subsubsection{Graphs for Jitter}

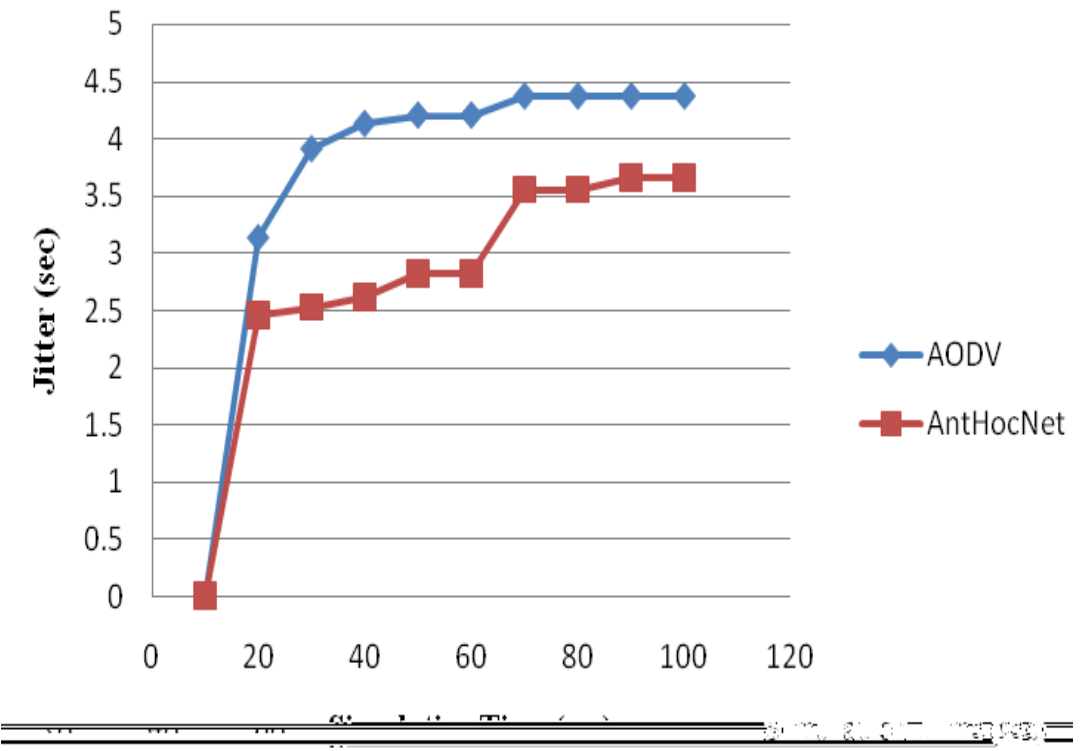

Figure 5. UDP Jitter at Data Rate 1Mbps

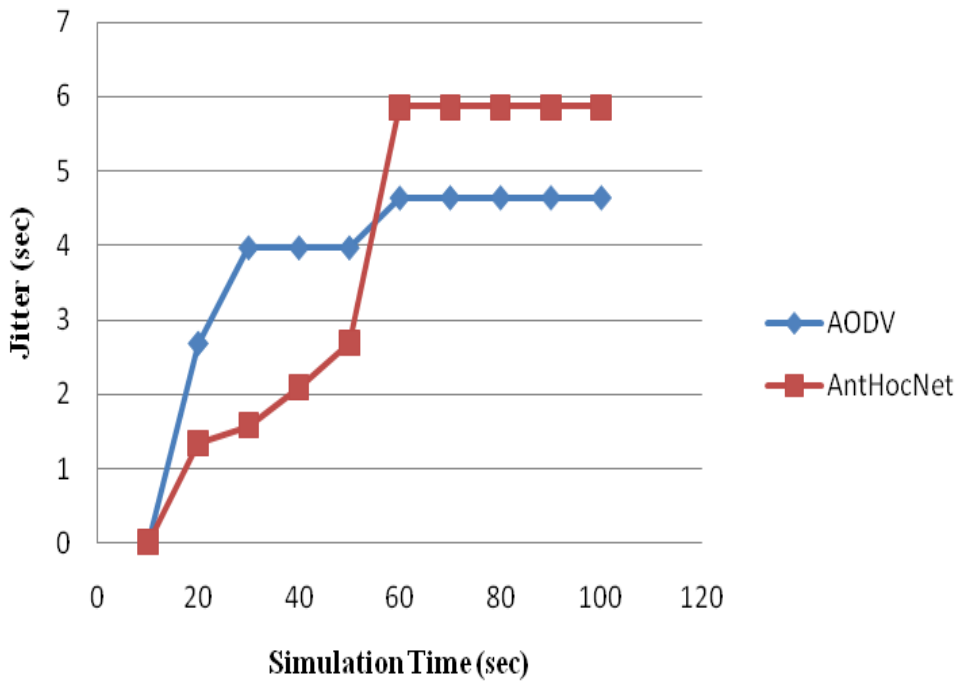

Figure 6. UDP Jitter at Data Rate 4Mbps 
International Journal on Cybernetics \& Informatics (IJCI) Vol. 4, No. 2, April 2015

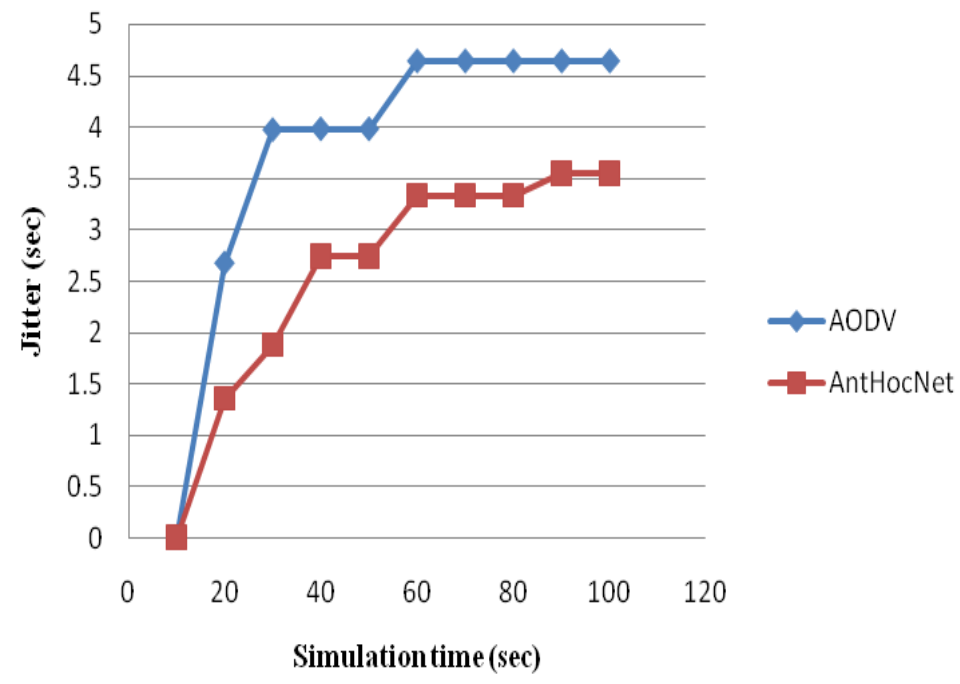

Figure 7. UDP Jitter at Data Rate $8 \mathrm{Mbps}$

\subsubsection{Case 2: $802.11 b$}

Table 4. UDP Loss Rate with variation in data rates

\begin{tabular}{|l|l|l|}
\hline \multirow{2}{*}{$\begin{array}{l}\text { Data Rate } \\
(\text { Mbps })\end{array}$} & \multicolumn{1}{|l|}{ Loss Rate } \\
\cline { 2 - 3 } AODV & \multicolumn{1}{|l|}{ AntHocNet } \\
\hline 1 & 0.85 & 0.846 \\
\hline 2 & 0.92 & 0.926 \\
\hline 3 & 0.94 & 0.949 \\
\hline 4 & 0.96 & 0.962 \\
\hline 5 & 0.97 & 0.97 \\
\hline 6 & 0.97 & 0.99 \\
\hline 8 & 0.98 & 0.983 \\
\hline 9 & 0.98 & 0.984 \\
\hline
\end{tabular}

Table 5.UDP PDR with variation in data rates

\begin{tabular}{|l|l|l|}
\hline \multirow{2}{*}{$\begin{array}{l}\text { Data Rate } \\
(\text { Mbps })\end{array}$} & \multicolumn{1}{|l|}{ POD } & \multicolumn{1}{|l|}{ AntHocNet } \\
\cline { 2 - 3 } & 0.153 & 0.154 \\
\hline 1 & 0.079 & 0.074 \\
\hline 3 & 0.053 & 0.05 \\
\hline 4 & 0.039 & 0.04 \\
\hline 5 & 0.032 & 0.03 \\
\hline 6 & 0.026 & 0.014 \\
\hline 8 & 0.019 & 0.017 \\
\hline 9 & 0.018 & 0.015 \\
\hline
\end{tabular}


International Journal on Cybernetics \& Informatics (IJCI) Vol. 4, No. 2, April 2015

Table 6. UDP End -to- End delay with variation in data rates

\begin{tabular}{|l|l|l|}
\hline \multirow{2}{*}{$\begin{array}{l}\text { Data Rate } \\
\text { (Mbps) }\end{array}$} & \multicolumn{2}{|l|}{ End-End delay (sec) } \\
\cline { 2 - 3 } AODV & AntHocNet \\
\hline 1 & 0.808 & 0.821 \\
\hline 2 & 0.789 & 0.8004 \\
\hline 3 & 0.719 & 0.814 \\
\hline 4 & 0.789 & 0.824 \\
\hline 5 & 0.789 & 0.793 \\
\hline 6 & 0.789 & 0.473 \\
\hline 8 & 0.789 & 0.749 \\
\hline 9 & 0.807 & 0.813 \\
\hline
\end{tabular}

\subsubsection{Graphs for Throughput}

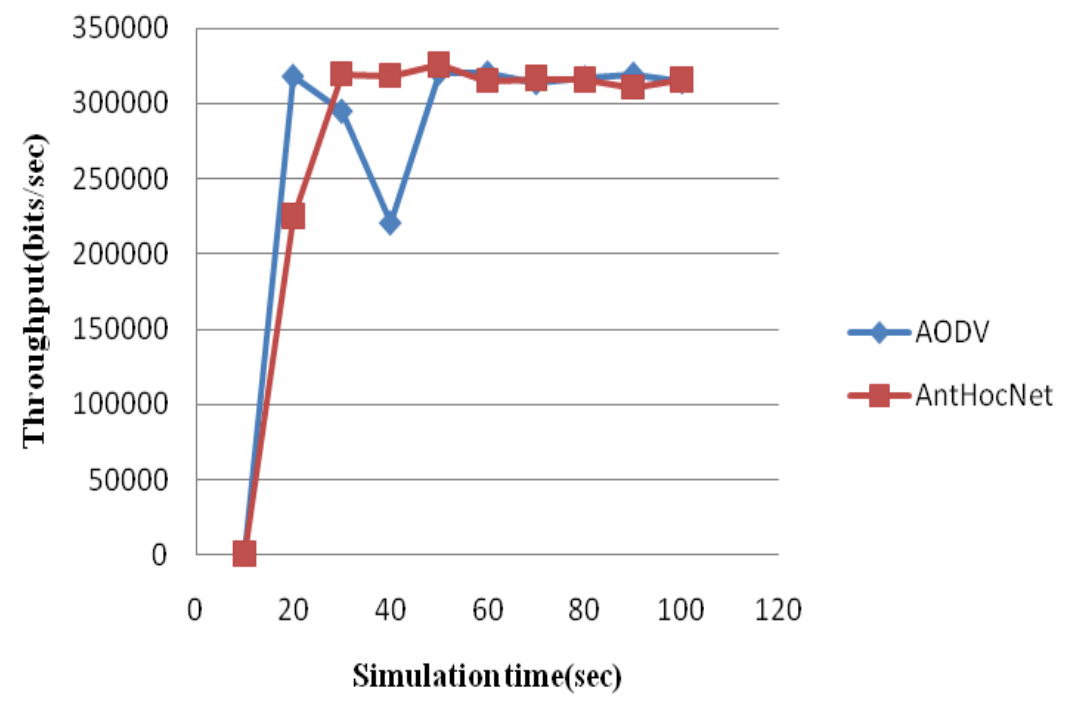

Figure 8. UDP Throughput at Data Rate 1Mbps

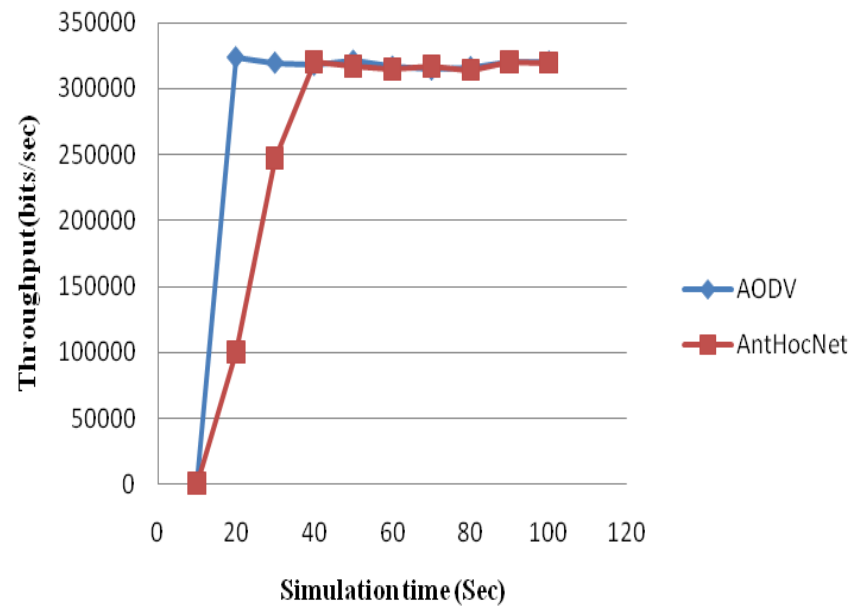

Figure 9. UDP Throughput at Data Rate 5Mbps 
International Journal on Cybernetics \& Informatics (IJCI) Vol. 4, No. 2, April 2015

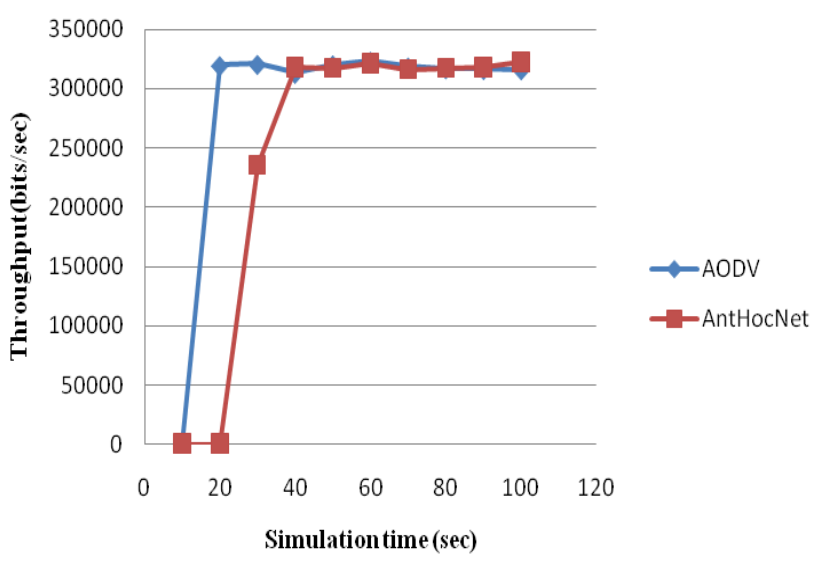

Figure 10. UDP Throughput at Data Rate 9Mbps

\subsubsection{Graphs for Jitter}
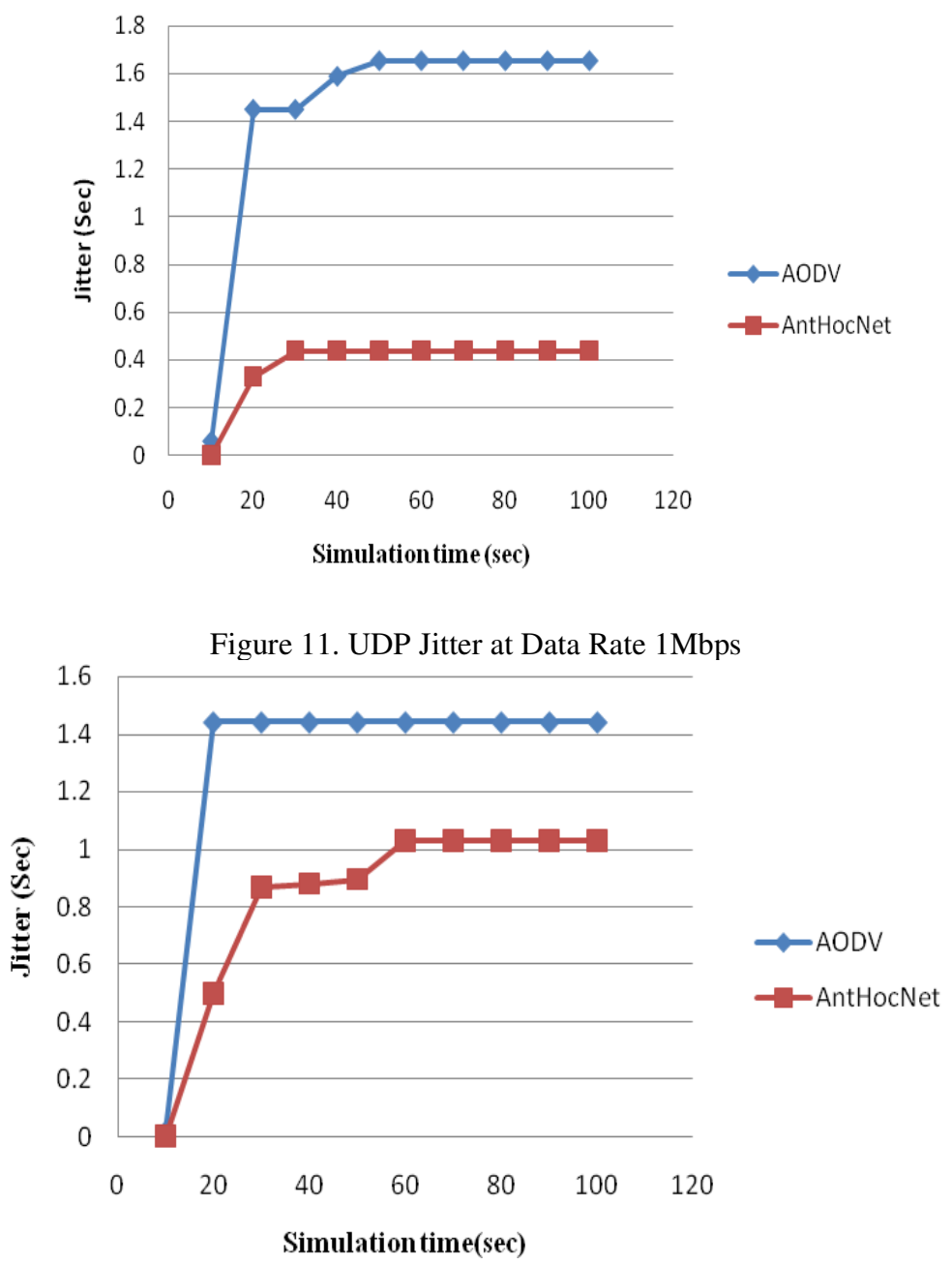

Figure 12. UDP Jitter at Data Rate 5Mbps 
International Journal on Cybernetics \& Informatics (IJCI) Vol. 4, No. 2, April 2015

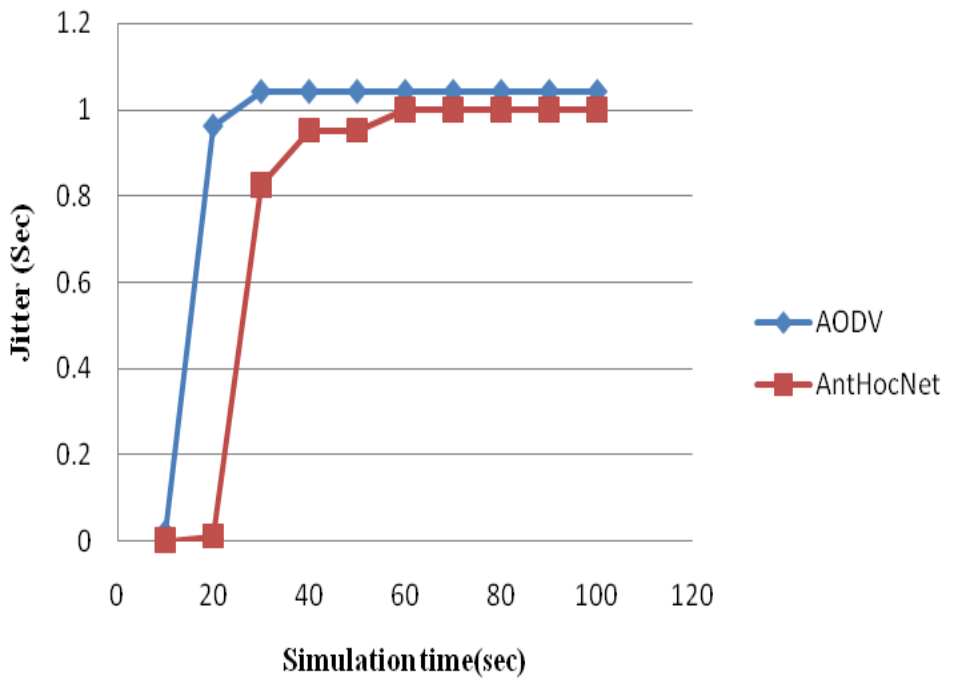

Figure 13. UDP Jitter at Data Rate 9Mbps

\section{DisCUSSION}

In case 1 simulations are done by using 802.11. Table 1 shows that evaluation of loss rate at different data rates. From the results it can be concluded that AntHocNet has lower performance than AODV. At different data rates AODV has high packet delivery ratio than AntHocNet, and is shown in Table 2. From Table 3, it is inferred that AntHocNet has lower performance than AODV in terms of end-to-end delay at distinct data rates.

The graphs represent the evaluation of throughput at different simulation times and different data rates. Figure 2 illustrates that AODV has high throughput than AntHocNet at data rate 1Mbps. Throughput of AODV is better than AntHocNet at data rate 4Mbps, and is shown in Figure 3. From the results obtained in Figure 4, it is concluded that AODV's throughput is superior to AntHocNet at $8 \mathrm{Mbps}$. From Figures 5, 6 and 7 it is concluded that AntHocNet has better performance than AODV in case of jitter.

In case 2 simulations are performed by using $802.11 \mathrm{~b}$. From Table 4 , it is concluded that AntHocNet has lower performance than AODV in case of Loss Rate. In Table 5, simulation results show that AODV has high performance than AntHocNet in terms of Packet Delivery Ratio. Table 6 refers that AntHocNet has less End-to-End Delay than AODV.

In Figure 8, Throughput is evaluated at various simulation times and data rate $1 \mathrm{Mbps}$. Here throughput is best for AODV. At data rate $5 \mathrm{Mbps}$ and different simulation times AODV Throughput is higher than AntHocNet as shown Figure 9. Figure 10 represents Throughput at various simulation times and data rate $9 \mathrm{Mbps}$, and from the results it is concluded that AODV performance is superior to AntHocNet. The Jitter is evaluated at various simulation times and different data rates $1 \mathrm{Mbps}, 5 \mathrm{Mbps}$ and $9 \mathrm{Mbps}$ as shown in Figures 11, 12, 13. It is concluded that the AntHocNet exhibits better performance than AODV.

\section{CONCLUSiON}

Simulation results conclude that with IEEE 802.11, AODV protocol performs better than AntHocNet protocol in case of metrics Loss Rate, End-to-End Delay, Packet Delivery Ratio and 
Throughput, whereas AntHocNet performs better than AODV protocol when the metric is Jitter. Simulation results conclude that with IEEE 802.11b, AntHocNet performs better than AODV in case of End-to-End Delay and remaining results are similar with IEEE 802.11.

\section{REFERENCES}

[1] S.B. Wankhade, M.S. Ali, (2012) "Recent Trends in Ant-Based Routing Protocols for MANET", International Journal of Advances in Engineering \& Technology, Vol. 4, Issue 1, pp. 405-413, ISSN: 2231-1963.

[2] Frederick Ducatelle, (2007) “Adaptive Routing in Ad-hoc Wireless Multi-hop Networks”, Lugano, Switzerland.

[3] Gianni Di Caro, Frederick Ducatelle, Luca Maria Gambardella, (2005) "Special Issue on Selforganization in Mobile Networking AntHocNet: An Adaptive Nature-inspired Algorithm for Routing in Mobile Ad-hoc Networks", European Transactions on Telecommunications, pp 16:443-455, DOI: 10.1002/ett.1062.

[4] Maahi Amit Khemchandani, Prof. B. W. Balkhande, (2014) "Comparative Analysis of AntHocNet, AODV, DSR Routing Protocols for Improvising Loss Packet Delivery Factor", International Journal of Computer Science and Information Technologies, Vol. 5 (5), pp 6478-6482, ISSN: 0975-9646.

[5] Annapurna P Patil, K Rajanikant, Rakshith H P, (2012) "Analyzing the Performance of AntHocNet Protocol for MANETS”, International Journal of Computer Applications, Vol. 57-No.5, pp 09758887, DOI: $10.5120 / 9111-3265$.

[6] Ahmed M. Abd Elmoniem, Hosny M. Ibrahim, Marghny H. Mohamed, Abdel-Rahman Hedar, (2012) "Ant Colony and Load Balancing Optimizations for AODV Routing Protocol", International Journal of SensorNetworks and Data Communications, Vol. 1, pp 14, DOI: 10.4303/ijsndc/X110203.

[7] D. Sivakumar, B. Suganthi, (2014) "Performance Comparison and Evaluation of Ad Hoc Routing Protocols", International Journal of Scientific \& Engineering Research, Vol. 5, Issue 4, ISSN: 22295518.

[8] Nishitha Taraka, Amarnath Emani, (2014) "Routing in Ad Hoc Networks Using Ant Colony Optimization", IEEE $5^{\text {th }}$ International Conference on Intelligent Systems, Modelling and Simulation, Langkawi (Malaysia), pp 2166-0662/14, DOI: 10.1109/ISMS.2014.100.

[9] N.S. Labhade, S. S. Vasekar, (2014) "Comparison of Proactive, Reactive and Hybrid Routing Protocols in Mobile Ad- Hoc Network", International Journal of Innovative Research \& Studies, Vol. 3, Issue 6, ISSN 2319-9725. 